Pushing the limits of plasma length in inertial fusion laser-plasma interaction experiments

D. Froula, L. Divol, R. London, P. Michel, R. L. Berger, N. Meezan, P. Neumayer, J. Ross, R.J. Wallace, S. H. Glenzer

August 13, 2007

Pushing the limits of plasma length in inertial fusion laser-plasma interaction experiments 
This document was prepared as an account of work sponsored by an agency of the United States Government. Neither the United States Government nor the University of California nor any of their employees, makes any warranty, express or implied, or assumes any legal liability or responsibility for the accuracy, completeness, or usefulness of any information, apparatus, product, or process disclosed, or represents that its use would not infringe privately owned rights. Reference herein to any specific commercial product, process, or service by trade name, trademark, manufacturer, or otherwise, does not necessarily constitute or imply its endorsement, recommendation, or favoring by the United States Government or the University of California. The views and opinions of authors expressed herein do not necessarily state or reflect those of the United States Government or the University of California, and shall not be used for advertising or product endorsement purposes. 


\title{
Pushing the limits of plasma length in inertial fusion laser-plasma interaction experiments
}

\author{
D. H. Froula, ${ }^{*}$ L. Divol, R. A. London, P. Michel, R. L. Berger, N. \\ B. Meezan, P. Neumayer, J. S. Ross, R. Wallace, and S. H. Glenzer \\ L-399, Lawrence Livermore National Laboratory, P.O. Box 808, Livermore, CA 94551, USA
}

(Dated: August 10, 2007)

\begin{abstract}
We demonstrate laser beam propagation and low backscatter in laser produced hohlraum plasmas of ignition plasma length. At intensities $I<5 \times 10^{14} \mathrm{~W}-\mathrm{cm}^{-2}$ a blue $(3 \omega, 351 \mathrm{~nm})$ laser beam propagates greater than $80 \%$ of its energy through a $\mathrm{L}=5$-mm long, high temperature $\left(\mathrm{T}_{e}=2.5 \mathrm{keV}\right)$, high density $\left(\mathrm{n}_{e}=5 \times 10^{20} \mathrm{~cm}^{-3}\right)$ plasma. These experiments show that the backscatter scales exponentially with plasma length as consistent with linear theory. The backscatter calculated by a new steady state 3D laser-plasma interaction code developed for large ignition plasmas is in good agreement with the measurements.
\end{abstract}

PACS numbers: $52.25 . \mathrm{Os}, 52.35 . \mathrm{Fp}, 52.50 . \mathrm{Jm}$

Keywords: laser plasma, laser beam propagation, transmitted beam diagnostic

The next generation of lasers for high energy density science will produce long pulse lengths (>10 ns) at high power, generating large scale plasmas. In the indirect drive approach to inertial confinement fusion (ICF), laser beams will propagate through centimeter scale underdense $\left(n_{e} \simeq 5 \times 10^{20} \mathrm{~cm}^{-3}\right)$ plasmas prior to producing soft $x$-ray radiation near the inner wall of the radiation cavity (hohlraum) [1, 2]. The radiation environment is designed to spherically compress a capsule containing nuclear fuel to fusion conditions. The inside of the hohlraum will be filled with a low-Z, long-scale length [3], high-temperature plasma consisting of the initial gas fill, and material ablated from the capsule and the inner hohlraum surfaces. Efficient laser beam propagation through this long plasma is required for controlling $\mathrm{x}$-ray conversion and radiation symmetry.

Laser-plasma interactions in long scale length plasmas may resonantly drive plasma waves that can backscatter the incident laser light $[4,5]$. Linear theory predicts that these scattering processes will grow exponentially from thermal noise with the length (L) and laser beam intensity (I) as evident in the linear gain exponent $\left(G_{s b s}\right)$ for stimulated Brillouin scattering (SBS): $G_{s b s} \propto \operatorname{ILn}_{e} / T_{e}$.

Recent research has focused on accurately verifying this scaling law by varying the laser beam intensity (I) $[6,7]$, the electron density $\left(n_{e}\right)[8]$ and temperature $\left(T_{e}\right)[9]$ in laser created plasmas that are limited in length to $L<2 \mathrm{~mm}$. Designs for ICF targets aim at lowering the gain exponent by increasing the laser spot size to reduce the intensity, controlling the electron density, and reaching multi-keV electron temperatures $[10,11]$. For centimeter long ICF targets, this approach relies on the length scaling predicted by linear theory. Scaling the plasma length beyond $2 \mathrm{~mm}$ challenges both the current state-of-the-art modeling and experimental facilities through computational requirements to resolve micron scales over many cubic millimeters of plasma and laser power to heat the large $(\mathrm{L}=5 \mathrm{~mm})$ plasmas to relevant

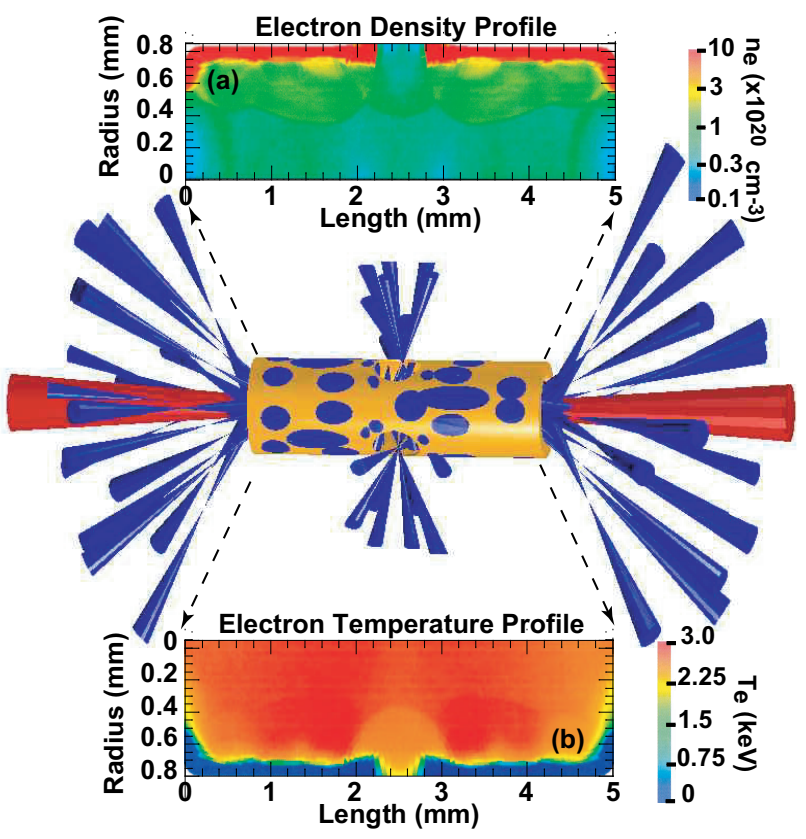

FIG. 1: The simulated (a) electron density and (b) electron temperature profiles are shown for the 5 -mm long hohlraum at peak electron temperature (900 ps after the heater beams turn on). Three LEH are equally spaced around the hohlraum equator to allow the addition of 17 laser beams. In total, fifty $3 \omega$ laser beams irradiate the hohlraum wall, producing electron temperature along the axis in excess of $T_{e}>2.5 \mathrm{keV}$. The interaction beam is aligned along the axis of the hohlraum interacting with a uniform $5 \times 10^{20} \mathrm{~cm}^{-3}$ plasma plateau.

temperatures $\left(T_{e}>2.5 \mathrm{keV}\right)$.

In this study, we present the first scaling of laserplasma interactions in ICF conditions (no filamentation) with plasma lengths (2-mm to 5 - $\mathrm{mm}$ ) approaching ignition hohlraum conditions. These experiments show that the backscatter scales exponentially with the plasma length, increasing by three orders of magnitude when in- 

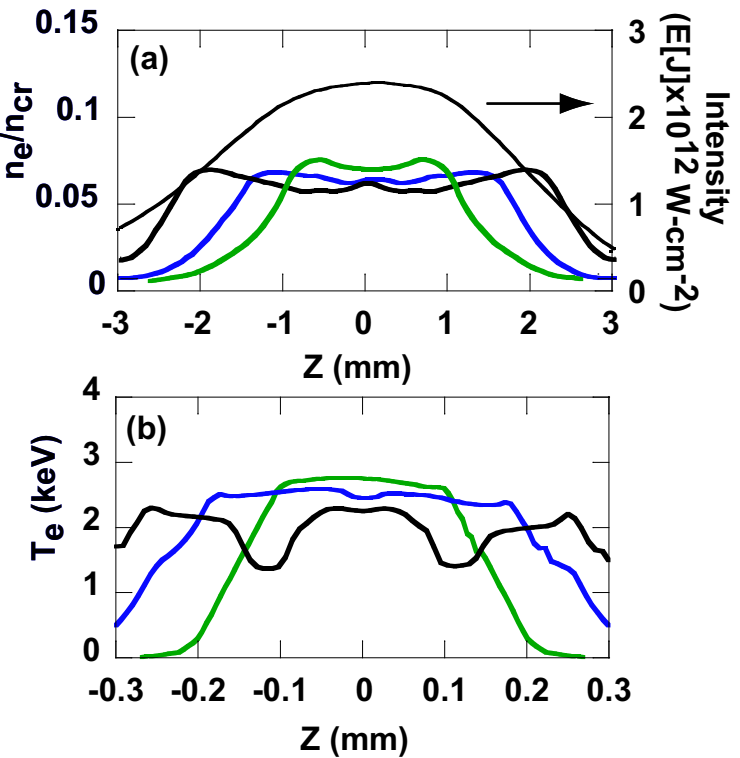

FIG. 2: (color) (a) The calculated electron density (left axis) along the interaction beam path $(\mathrm{Z})$ is plotted for $2-\mathrm{mm}$ (green), 3.5-mm (blue), 5-mm (black) long hohlraums. The interaction beam vacuum intensity profile along the hohlraum axis is shown in black (right axis). (b) The electron temperature along the interaction beam path is plotted for $2-\mathrm{mm}$ (green), 3.5-mm (blue), and 5-mm (black) long hohlraums. The profiles are averaged over the diameter of the interaction beam 700 ps after the heater beams turn on.

creasing the length of the plasma from $1.8 \mathrm{~mm}$ to $3.9 \mathrm{~mm}$. The intensity threshold defined at $R=5 \%$ is measured to be $\mathrm{I}=6 \times 10^{14} \mathrm{~W}-\mathrm{cm}^{-2}$ for propagation through this 5 $\mathrm{mm}$ target while the threshold was increased in our 2-mm target to $\mathrm{I}=13 \times 10^{14} \mathrm{~W}-\mathrm{cm}^{-2}$. Increasing the intensity significantly above these thresholds results in saturation due to pump depletion. The results compare well with a new laser-plasma interaction code (SLIP) [12] designed to simulate long 3D plasmas with large diameter laser beams encountered in the future ignition experiments.

For this study, new hohlraum targets have been designed for investigating laser-plasma interactions in 2$\mathrm{mm}, 3.5-\mathrm{mm}$, and 5-mm long high-temperature plasmas (Fig. 1). An interaction beam is directed down the axis of a gas-filled gold hohlraum. This allows direct measurements of the laser beam propagation and transmission through ignition-scale hohlraum plasmas. The three gold hohlraums have the same diameter $(1.6 \mathrm{~mm})$ and produce a uniform density plateau using a room temperature $1 \mathrm{~atm}$ gas fill consisting of $30 \% \mathrm{CH}_{4}$ and $70 \% \mathrm{C}_{3} \mathrm{H}_{8}$ expressed as partial pressures. The results reported here are performed over a time when there exists a uniform density plateau (Fig. 2) before the blast waves driven by laser beam heating of the gold wall reach the hohlraum axis.

Experiments were performed at the Omega Laser Facility [13]. For all targets, thirty-three frequency tripled

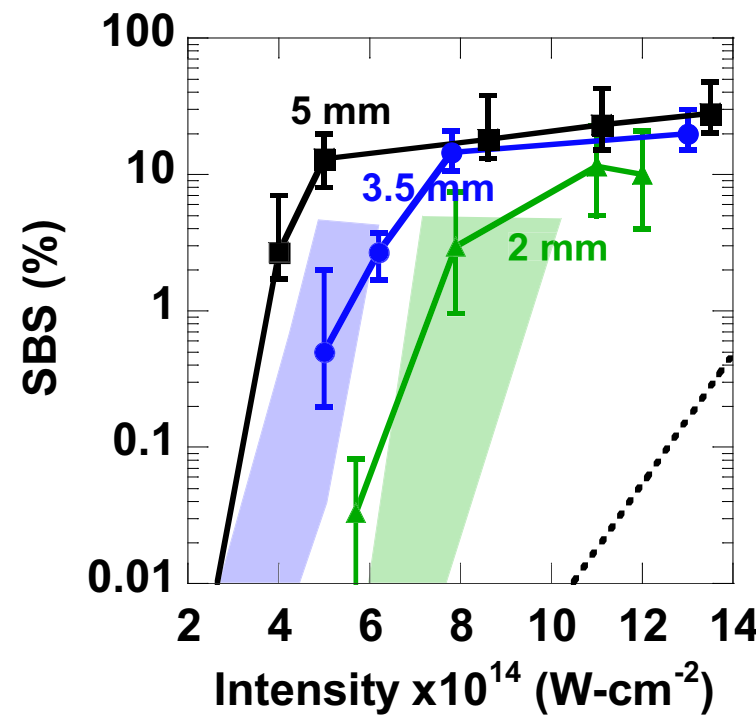

FIG. 3: (color) The measured instantaneous SBS reflectivity is plotted as a function of vacuum intensity for the three target lengths: $2 \mathrm{~mm}$ (triangles), $3.5 \mathrm{~mm}$ (circles), and $5 \mathrm{~mm}$ (squares). The shaded regions represent simulations for the 2$\mathrm{mm}$ and $3.5-\mathrm{mm}$ long targets. The boundaries are given by including (left side) and not including (right side) filamentation in the simulation. For reference, a curve is shown (dashed) calculated using linear theory for the 2-mm long targets where a gain of $G_{s b s}=11$ is determined by post processing the hydrodynamic parameters.

$\left(\lambda_{o}=0.351 \mu \mathrm{m}\right)$ laser beams enter through 800 micron diameter laser entrance holes (LEHs) at the ends of the hohlraum while the $3.5-\mathrm{mm}$ and 5 - $\mathrm{mm}$ targets used an additional 17 laser beams entering through three equally spaced 1200 micron diameter LEH around the equator of the hohlraum (Fig. 1). The laser beams were configured with $480 \mathrm{~J} /$ beam in a 1 -ns flat pulse $(+0.15 \mathrm{~ns}$ rise and fall).

Previous studies have characterized the 2-mm long target platform along the interaction beam path using Thomson scattering. The peak electron temperature was measured to be $T_{e}=3.5 \mathrm{keV}$ [14]. To maintain high temperatures as the hohlraum is lengthened, the laser heater beam power must be increased. The hydrodynamic simulations exhibit a large region of gas and blowoff plasma inside the hohlraum that is nearly isothermal [see Fig. 1(b)]. The temperature of the isothermal region is controlled by a balance of laser heating with increasing thermal energy, radiation cooling, and electron heat transfer to the cold gold walls. The energy loss scales approximately linearly with the hohlraum length, since the loss to the LEHs and to the hohlraum end caps is fairly small. Therefore, maintaining a constant laser power per unit length keeps the temperature approximately constant as the length is increased.

The addition of 17 heater beams that enter through the LEHs on the equator of the hohlraum allows us to keep 



FIG. 4: (color) The SBS reflectivity as a function of wavelength is shown for the three target platforms (a) $2 \mathrm{~mm}$, (b) $3.5 \mathrm{~mm}$, and (c) $5.0 \mathrm{~mm}$ measured at a vacuum intensity of $1.2 \times 10^{15} \mathrm{~W}-\mathrm{cm}^{-2}$. (d) The synthetic SBS reflectivity spectrum is shown for the 5-mm target and is generated by post processing the hydrodynamic simulations using LIP and linear theory including pump depletion. The good agreement between these spectra confirms that SBS comes from the central density plateau. The wavelength $\operatorname{shift}(\Delta \lambda=8.5 \AA)$ at $700 \mathrm{ps}$ is consistent with the simulated electron temperature $\left(T_{e}=2.4 \mathrm{keV}\right)$.

the total laser power per unit length roughly constant between the 2-mm and 3.5 -mm targets; this produces hot $T_{e}>2.5 \mathrm{keV}$ plasmas for all three targets platforms.

Light scattered from the interaction beam is measured using a full-aperture backscatter station (FABS), near backscatter imager (NBI), and a $3 \omega$ transmitted beam

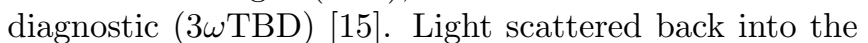
original beam cone is collected by the FABS; both stimulated Brillouin scattering (SBS) and stimulated Raman scattering (SRS) spectra and energies are independently measured. The NBI measures backscattered light outside the original beam cone that reflects from a plate surrounding the interaction beam. The plate is imaged onto two charge-coupled devices (CCD) which time integrate the SBS and SRS signals. In this study, a new calibration technique was employed using a pulsed calibration system to deliver a known power to the NBI scatter plate and the FABS calorimeters. The uncertainty in the measurements of the total SBS energy using this system is $15 \%$. The $3 \omega \mathrm{TBD}$ allows us to accurately measure the interaction beam power after propagating through the plasmas. In addition, the transmitted energy, spectrum, and temporal beam spray are recorded. The system collects the light forward scattered within twice the original $f / 6.7$ beam cone.

Figure $2 \mathrm{a}$ shows the vacuum intensity profile for the $3 \omega$ interaction beam as it propagates along the hohlraum axis. The beam is focused by a $f / 6.7$ lens through a continuous phase plate (CPP)[16] to a minimum vacuum diameter of 200 microns at the center of the hohlraum. For the $3.5-\mathrm{mm}$ and $5-\mathrm{mm}$ targets, the interaction beam ( 1 ns square pulse) is delayed relative to the heater beams by $0.5 \mathrm{~ns}$, while a delay of $0.3 \mathrm{~ns}$ is used for the $2-\mathrm{mm}$ targets.

Figure 3 demonstrates a clear plasma length scaling for various interaction beam intensities. Extending the plasma length from $1.8 \mathrm{~mm}$ to $3.9 \mathrm{~mm}$ reduces the intensity threshold from $\mathrm{I}_{2 \mathrm{~mm}}=13 \times 10^{14} \mathrm{~W}-\mathrm{cm}^{-2}$ to $\mathrm{I}_{5 \mathrm{~mm}}=6 \times 10^{14} \mathrm{~W}-\mathrm{cm}^{-2}$ as predicted by scaling of linear theory. The data points in Fig. 3 are obtained at a time 700 ps after the rise of the heater beams and averaged over 50 ps. The error bars are given by the upper and lower limits within this average. No SRS $\left(R_{\text {srs }}<1 \%\right)$ is measured in these experiments while the heater beams are on (up to $1.1 \mathrm{~ns}$ ), as predicted by the moderate linear SRS gains $\left(G_{\text {srs }}<10\right)$.

The simulated SBS thresholds shown in Fig. 3 are in good agreement with the measurements. These thresholds were calculated by a recently implemented steady state paraxial coupled-wave-solver (SLIP) [12]. SLIP solves the linear kinetic model for SBS using the complete 3-dimensional plasma parameters, the measured near field of the interaction beam, and near-field phase induced by the CPP. The ability of SLIP to include the 3dimensional nature of the spatially smoothed laser beam is a significant improvement over the standard 1D linear gain calculations (LIP) that are limited to geometric ray propagation [17]. This is evident in Fig. 3, where the linear gain calculations significantly under-estimate the backscatter. Near the threshold, SLIP calculates the most intense speckles within the laser focal spot to trigger SBS locally while the average intensity used in LIP is still under the threshold. Even when the backscatter from these high intensity speckles remain small, they can act as increased noise source and trigger SBS for the whole beam, making the $1 \mathrm{D}$ model inaccurate.

The 3-dimensional plasma parameters used in the SLIP calculations were simulated by the hydrodynamic code HYDRA [18]. The plasma parameters are calculated as a function of time in 2-dimensions using the experimental 
laser beam configurations. For the long scale targets, a ring is cut out of the hohlraum to allow laser beams to enter on the equator. Figure 1 shows the 2D simulated electron densities and temperatures for the 5-mm targets used as input for the SLIP calculations. These plasma parameters are calculated using a Spitzer-Härm electron conduction model with a flux limited $f=0.05$. The plasma parameters along the interaction beam (Fig. 2) have been shown to be insensitive to the heat conduction models for the 2-mm long targets [14].

Figure 4 compares the SBS spectrum measured from the 2-mm, 3.5-mm, and 5-mm long targets. Backscatter from the 2-mm long target decreases with time below detectable limits as the linear gain exponent is reduced due to an increasing electron temperature [9]. For plasma lengths larger than $3.5 \mathrm{~mm}$, the backscatter is maintained until the hohlraum closes due to stagnation (up to $1.1 \mathrm{~ns}$ ) despite the increasing electron temperature. All three target platforms show a narrow spectral feature upshifted by $\sim 0.85 \mathrm{~nm}$ from the fundamental wavelength of the laser at peak electron temperature. This finding is well reproduced by our linear gain calculations. Figure 4(d) shows a simulated SBS spectrum for the 5-mm target obtained by postprocessing the linear gains calculated by LIP with linear theory including pump depletion [11, 19]. The large wavelength shift and narrow spectral features are direct evidence of the high electron temperatures $\left(T_{e}>2.5 \mathrm{keV}\right)$ and scattering from the uniform plasma conditions in the long plasma plateaus.

Figure 5 presents the temporal evolution of the SBS reflectivity for the longest target. The SBS reflectivity peaks early in time when the plasma temperature is low. For an intensity of $1 \times 10^{15} \mathrm{~W}-\mathrm{cm}^{-2}$, the linear gain exponent calculated by post-processing the hydrodynamic simulations using LIP [10], peaks during the rise of the interaction beam $\left(G_{s b s}=28\right)$ and decreases to a nearly constant plateau $\left(G_{s b s}=19\right)$ by the middle of the interaction beam. For intensities less than $5 \times 10^{14} \mathrm{~W}-\mathrm{cm}^{-2}$, the 5 -mm long plasma is transparent: we measure greater than $80 \%$ transmission at peak electron temperature. Inverse Bremsstrahlung calculations agree well with these measurements and show a transmission above $90 \%$ when the temperature exceeds $T_{e}>2.25 \mathrm{keV}$. When the interaction beam intensity is above $1 \times 10^{15} \mathrm{~W}-\mathrm{cm}^{-2}$, the peak transmission is reduced to $40 \%$ due to backscatter.

In summary, we have demonstrated that stimulated Brillouin scattering scales with plasma-length as predicted by linear theory for high-temperature fusion plasma conditions. The backscatter is measured to increase by three orders of magnitude when increasing the length of the plasma from $2 \mathrm{~mm}$ to $5 \mathrm{~mm}$. The SBS threshold is reduced by a factor of 2 when the length is increased by the same factor. These experiments represent ignition relevant conditions justifying the extrapolation of the large data base of existing laser-plasma interaction

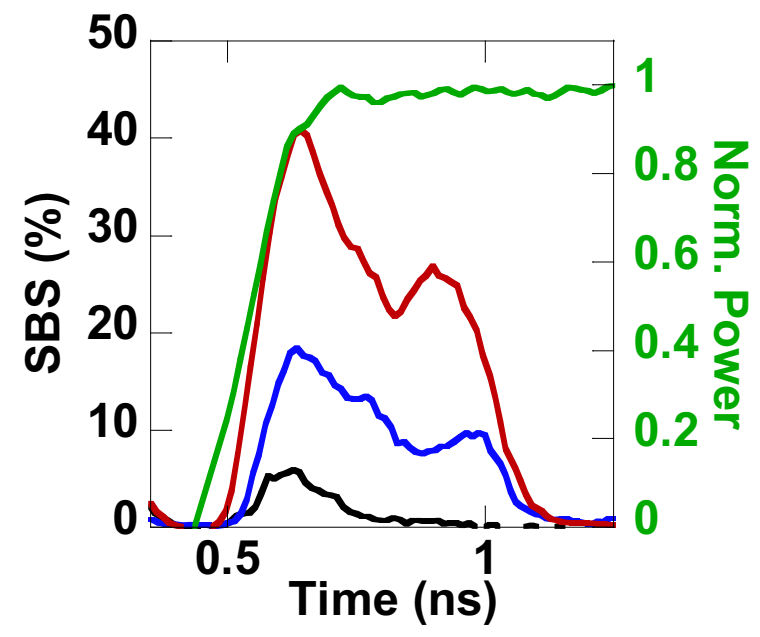

FIG. 5: (color) The SBS reflectivity as a function of time is shown for the longest plasma conditions (5-mm) measured at three intensities (red) $12 \times 10^{14} \mathrm{~W}-\mathrm{cm}^{-3}$, (blue) $8 \times 10^{14} \mathrm{~W}-\mathrm{cm}^{-3}$, (black) $5 \times 10^{14} \mathrm{~W}-\mathrm{cm}^{-3}$. The normalized power for the interaction beam is shown (right axis).

results to ignition relevant scales [6-9].

We would like to acknowledge the efforts of the Omega Laser Crew in fielding these challenging experiments. This work was supported by LDRD 06-ERD-056 and performed under the auspices of the U.S. Department of Energy by the Lawrence Livermore National Laboratory under Contract No. W-7405-ENG-48.

* Electronic address: froula1@llnl.gov

[1] J. D. Lindl et al., Phys. Plasmas 11, 339 (2004).

[2] R. P. Drake, High-Energy-Density Physics (Springer Berlin Heidelberg, New York, 2006).

[3] S. H. Glenzer et al., Nature Physics Accepted (2007).

[4] W. L. Kruer, The Physics of laser plasma interactions (Addison-Wesley Publishing Company, Inc., 1988).

[5] H. Baldis et al., Phys. Rev. Lett. 80, 1900 (1998).

[6] B. Macgowan et al., Phys. Plasmas 3, 2029 (1996).

[7] W. Seka et al., Phys. Rev. Lett. 89, 175002 (2002).

[8] D. S. Montgomery et al., Phys. Plasmas 3, 1728 (1996).

[9] D. H. Froula et al., Phys. Rev. Lett. 98, 085001 (2007).

[10] N. B. Meezan et al., Phys. Plasmas 14, 056304 (2007).

[11] D. H. Froula et al., Phys. Plasmas 14, 055705 (2007).

[12] P. Michel and L. Divol, To be submitted to Phys. Plasmas (2007).

[13] J. M. Soures et al., Laser Particle Beams 11, 317 (1993).

[14] D. Froula et al., Phys. Plasmas 13, 052704 (2006).

[15] D. H. Froula et al., Rev. Sci. Instrum. 77, 10E507 (2006).

[16] S. Dixit et al., Opt. Lett. 21, 1715 (1996).

[17] E. A. Williams, Tech. Rep. UCRL-LR-105820-98, Lawrence Livermore National Laboratory (1998).

[18] M. M. Marinak et al., Phys. Plasmas 8, 2275 (2001).

[19] C. Tang, J. Appl. Phys. 37, 2945 (1966). 